\title{
Trigeminal Nerve Schwannoma of the Cerebellopontine Angle
}

\author{
Maria Peris-Celda ${ }^{1}$ Christopher S. Graffeo ${ }^{1}$ Avital Perry ${ }^{1}$ Lucas P. Carlstrom ${ }^{1}$ Michael J. Link ${ }^{1,2}$ \\ ${ }^{1}$ Department of Neurologic Surgery, Mayo Clinic, Rochester, \\ Minnesota, United States \\ 2 Department of Otolaryngology-Head and Neck Surgery, Mayo Clinic, \\ Rochester, Minnesota, United States \\ Address for correspondence Michael J. Link, MD, Department of \\ Neurologic Surgery, Mayo Clinic, 200 First Street South West, \\ Rochester, MN 55905, United States \\ (e-mail: link.michael@mayo.edu).
}

J Neurol Surg B 2018;79(suppl S5):S389-S390.

\begin{abstract}
Keywords

- trigeminal

- schwannoma

- cerebellopontine angle

- microsurgery

Introduction Large and even moderate sized, extra-axial cerebellopontine angle (CPA) tumors may fill this restricted space and distort the regional anatomy. It may be difficult to determine even with high resolution magnetic resonance imaging (MRI) if the tumor is dural-based, or what the nerve of origin is if a schwannoma. While clinical history and exam are helpful, they are not unequivocal, particularly since many patients present with a myriad of symptoms, or conversely an incidental finding. We present an atypical appearing, asymptomatic CPA tumor, ultimately identified at surgery to be a trigeminal schwannoma. Case History A 40-year-old man presented with new-onset seizure. MRI identified an incidental heterogeneously contrast-enhancing CPA lesion (-Fig. 1A-D). The tumor was centered on the internal auditory canal (IAC) with no tumor extension into Meckel's cave, IAC or jugular foramen. Audiometry demonstrated $10 \mathrm{db}$ of relative left-sided hearing loss with $100 \%$ word recognition. Physical examination was negative for focal neurologic deficits. A retrosigmoid craniotomy was performed and an extra-axial, yellow-hued mass was encountered and resected, which was ultimately confirmed to originate from the trigeminal nerve (-Video 1). Gross total resection was achieved, and the patient recovered from surgery with partial ipsilateral trigeminal sensory loss and no other new neurologic deficits.

Conclusion Pure CPA trigeminal schwannomas are rare, but should be considered in the differential for enhancing CPA lesions. Although, Meckel's cave involvement is frequently observed, it is not universal, and pure CPA schwannomas of all cranial nerves IV-XII have been reported in the literature.

The link to the video can be found at: https://youtu.be/AlodYCu70F8.
\end{abstract}

Conflict of Interest

None.

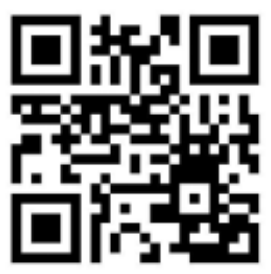

received

May 31, 2018

accepted

August 12, 2018

published online

September 25, 2018

www.thieme.com/skullbasevideos

www.thieme.com/jnlsbvideos

DOI https://doi.org/

10.1055/s-0038-1669966. ISSN 2193-6331.
๑) 2018 Georg Thieme Verlag KG
Stuttgart · New York

License terms

(c) (1) $\ominus$ (\$) 

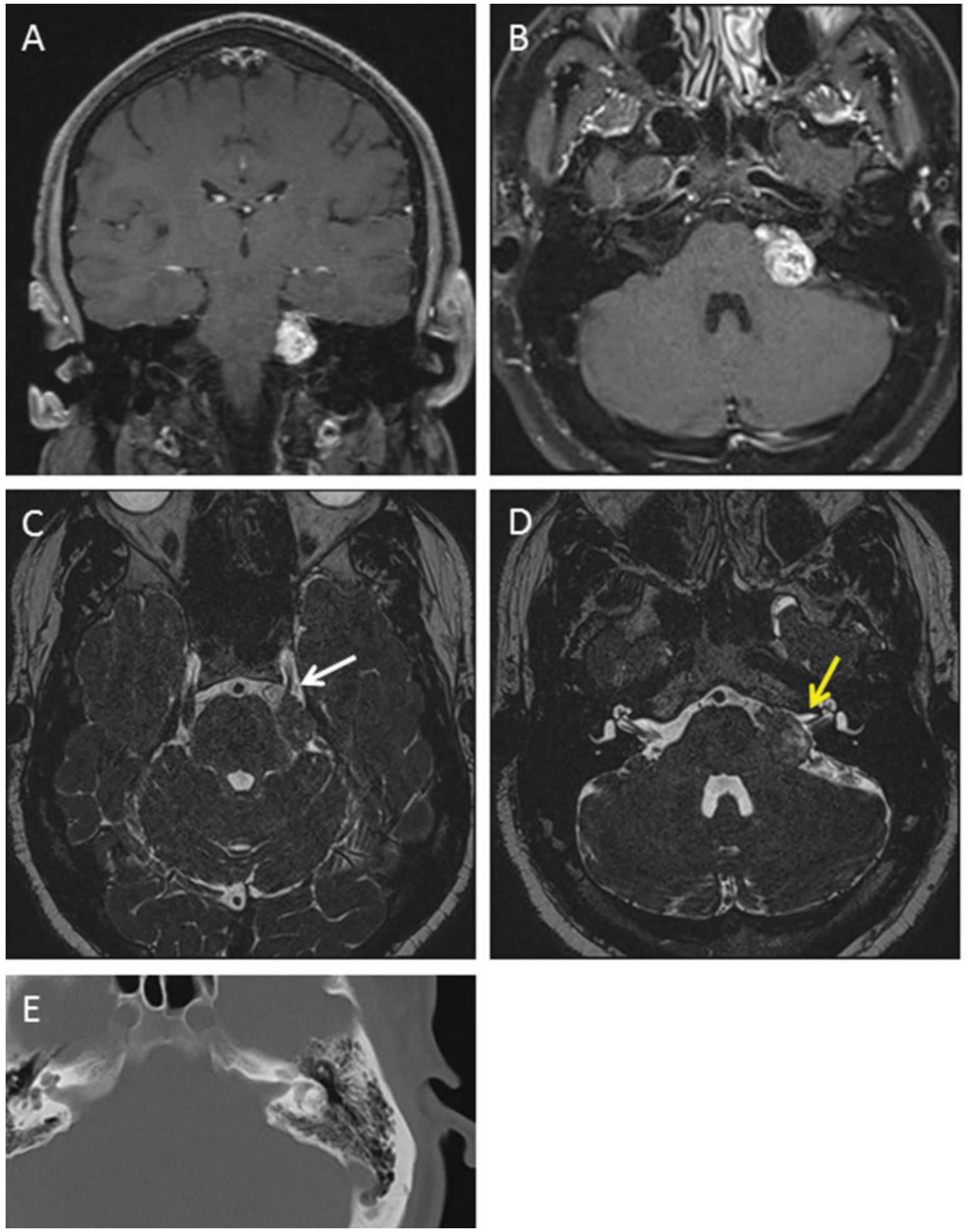

Fig. 1 (A-B) Coronal and axial T1 MRI sequences with contrast demonstrate an enhancing left CPA tumor. (C-D) Axial FIESTA images reveal no extension of the tumor into Meckel's cave (arrow) or the internal auditory canal (IAC; arrow). (E) Axial CT images show no evidence of bony erosion of the IAC. MRI, magnetic resonance imaging; CPA, cerebellopontine angle; FIESTA, fast imaging employing steady state acquisition; IAC, internal auditory canal; CT, computed tomography. 